\section{RMD Open}

Rheumatic \&

Musculoskeletal Diseases

To cite: Ding J, Eyre S, Worthington J. Genetics of RA susceptibility, what comes next?. RMD Open 2015;1:e000028. doi:10.1136/rmdopen-2014000028

- Prepublication history for this paper is available online. To view these files please visit the journal online (http://dx.doi.org/10.1136/ rmdopen-2014-000028).

Received 10 February 2015 Revised 25 March 2015 Accepted 28 March 2015

CrossMark

Arthritis Research UK Centre for Genetics and Genomics, Centre for Musculoskeletal Research, Institute of Inflammation and Repair, Manchester Academic Health Science Centre, The University of Manchester, Manchester, UK

Correspondence to Dr Jane Worthington; jane.worthington@ manchester.ac.uk

\title{
Genetics of RA susceptibility, what comes next?
}

James Ding, Stephen Eyre, Jane Worthington

\section{ABSTRACT}

Summary: Genome-wide association studies (GWASs) have been used to great effect to identify genetic susceptibility loci for complex disease. A series of GWAS and meta-analyses have informed the discovery of over 100 loci for rheumatoid arthritis (RA). In common with findings in other autoimmune diseases the lead signals for the majority of these loci do not map to known gene sequences. In order to realise the benefit of investment in GWAS studies it is vital we determine how disease associated alleles function to influence disease processes. This is leading to rapid development in our knowledge as to the function of non-coding regions of the genome. Here we consider possible functional mechanisms for intergenic RAassociated variants which lie within IncRNA sequences.

Rheumatoid arthritis (RA) is a polygenic autoimmune disease that is characterised by chronic inflammation of the synovial joints, and associated with the presence of anticitrullinated protein antibodies (ACPA). For over 40 years it has been well established that there is a strong association between susceptibility to RA and human leucocyte antigen (HLA) DRB1 alleles that share a consensus sequence, known as the 'shared epitope'. Spanning position 70-74 of the $\beta$ subunit of the HLA DR molecule, this association accounts for approximately $40 \%$ of the genetic component of susceptibility of RA, and has recently been refined following imputation of amino acid sequences from high-density single nucleotide polymorphism (SNP) data. ${ }^{2}$ It is only since the introduction of SNP-based genome-wide association studies (GWAS) in 2007 that an extensive list of additional genetic risk loci, have been associated with RA. ${ }^{3}$ Currently, over 100 risk loci have been defined, explaining approximately half of the genetic variance in RA. ${ }^{45}$

The challenge in this post-GWAS era is to interpret and translate these genetic findings, defining how they influence disease susceptibility and outcome. Signals from GWAS typically comprise large numbers of highly correlated SNPs defining regions that contain, on average, four genes, thus the remaining challenge is to identify for each locus the functional variant(s), the genes on which they act and the specific cell type in which dysregulation contributes to disease susceptibility.

In addition to providing insights into RA pathogenesis, understanding genetic susceptibility at this level has the potential to impact on both drug discovery and the optimal implementation of established therapies through a greater understanding of disease heterogeneity. Thus far, functional studies to further characterise implicated genetic regions in disease have focused almost exclusively on associated variants in protein-coding genes, however, these account for only a small minority of associated loci. Evidence suggests that for RA and many other complex diseases the majority of associated variants map outside protein coding regions.

\section{INTERGENIC RA LOCI}

In contrast to monogenic traits, an enrichment analysis performed using data from over 100 GWAS publications has shown that SNPs associated with complex traits are intergenic and do not map to protein sequences. ${ }^{6}$

Traditionally, the functional implications of intergenic RA loci have been attributed to the nearest protein-coding gene known to have immunological relevance. This assumption, that genetic polymorphisms alter the expression of neighbouring genes by affecting cis-regulatory elements, such as enhancers and promoters, has been demonstrated to be misguided at a number of loci. For example, an association between the chromosome $16 \mathrm{p} 13$ region and a number of autoimmune diseases has been shown. This association was originally attributed to CLEC16A, with the majority of disease associated SNPs found within the transcribed region of this gene, especially within intron 19. CLEC16A is expressed in a variety of immune relevant cells and contains an 
immunoreceptor tyrosine-based activation motif. Functional studies have, however, revealed a long range chromatin interaction between intron 19 of CLEC16A and regulatory sequences that affect the expression of DEXI, found $\sim 140 \mathrm{~kb}$ from the SNPs. ${ }^{7}$ The focus of research at this locus has now moved to DEXI as an autoimmune candidate gene. A similar example, where the SNPs in question are intergenic, rather than intronic, can be found at the chromosome 1 p13 region, which is associated with low-density lipoprotein cholesterol and myocardial infarction. Here a disease associated SNP disrupts a transcription factor binding site found between CELSR2 and PSRC1, which inhibits the expression of SORT1, found $\sim 35 \mathrm{~kb}$ from the putative causal SNP. ${ }^{8}$

Evidence is now emerging that the variants most strongly associated with autoimmune disease (lead SNPs) are enriched in areas of the chromosome that; are open and active, are defined by epigenetic marks, regulate gene transcription, and act in a cell-specific manner. Indeed, a recent study of 21 autoimmune diseases by Farh $e t a l^{9}{ }^{9}$ described an enrichment of putative causal SNPs in immune-cell enhancers. The majority $(\sim 60 \%)$ of these SNPs, associated with RA and 20 other autoimmune diseases, map to enhancer elements that are active in lymphoblasts, lymphocytes and macrophages. The RA-associated enhancers are enriched for; cell specificity ( $\mathrm{T}$ and $\mathrm{B}$ cells), activity in response to stimulation, and size (large 'super-enhancers'). Furthermore, they are associated with the expression of non-coding RNAs (enhancer-derived RNAs, eRNAs also known as elncRNAs). This was shown to be true within the subset of RA-associated super-enhancers in the analysis by Farh $e t a l$, but is best illustrated by Hnisz et $a l^{10}$ in one of the first papers to describe super-enhancers. Super-enhancers, consist of clusters of enhancer elements that play a key role in defining cell identity and are associated with a 24.3-fold enrichment of RNA, compared to typical enhancers. Fahr et al found a 3.2-fold enrichment of RA SNPs in super-enhancers compared to typical enhancers and speculated that these SNPs could elicit subtle and stimulation specific changes in gene expression. Defining the function of these ncRNA expressing regions will be pivotal if we are to translate GWAS findings into mechanisms of susceptibility.

By recruiting various proteins, enhancers mediate chromosomal interactions, enabling the assembly of transcriptional machinery at promoter regions. It is well established that these regulatory elements do not necessarily affect the transcription of neighbouring genes, can function over large distances and generally affect the expression of more than one gene. ${ }^{11}$ One of the hallmarks of these regulatory regions is accessible chromatin, as indicated by regions of DNase I hypersensitivity. Other markers that offer greater specificity include chromatin modifications: Enhancer regions are associated with high levels of histone 3 lysine 4 monomethylation and dimethylation, relative to trimethylation (H3K4me1, H3K4me2 and H3K4me3, respectively), with active enhancers characterised by histone 3 lysine 27 acetylation (H3K27ac). ${ }^{12}$

Lead disease-associated variants, situated as they are in gene regulatory regions, may have a number of functional consequences that contribute to increased risk of RA. These include disruption of transcription factor binding sites and chromatin interactions, as at the SORT1 and DEXI loci, as well as other mechanisms of disrupting regulatory features such as enhancers, silencers, promoters and insulators. Examples include potential impacts on the deposition of epigenetic markers and RNA binding. It is also possible for non-coding SNPs to affect the function of non-coding RNA transcripts, such as those of eRNA of lncRNA, by altering their sequence or expression pattern.

\section{LNCRNA}

lncRNA are traditionally defined by an arbitrary lower limit of 200 bases, with over 100000 transcripts identified in humans. ${ }^{13}$ Their diverse functions and characteristics have only recently attracted the attention of the scientific community (see box 1 for a description of various lncRNA categories). Further classification of lncRNA has focused on genomic content, with over a third of transcripts found in intergenic regions (long intervening non-coding RNA, lincRNA). While advances in RNA sequencing (RNA Seq) techniques can account for many of the IncRNA species identified to date, a large number of lincRNA have been identified by having a similar chromatin signature to that of other actively transcribed genes. Typically, trimethylation of histone 3 lysine 4 is (H3K4me3) is observed at the promoter, with histone 3 lysine 36 trimethylation (H3K36me3) found along the transcribed length.

lincRNAs are typically transcribed by RNA Polymerase II and often undergo polyadenylation and splicing, just like protein-coding transcripts. ${ }^{14}$ This is, however, not the case for some eRNAs, which are subdivided according to whether they are unidirectionally or bidirectionally transcribed (1D-eRNA, 2D-eRNA). eRNA are

\section{Box 1 Summary of IncRNA features and classification.}

Long non-coding RNA (IncRNA)

- Defined as over 200nt in length. Generally transcribed by RNA Polymerase II, spliced and polyadenylated, as for proteincoding genes.

- IncRNA are often characterised according to genomic position for example, exonic, intergenic (lincRNA), etc.

- Other, functional categories have been proposed.

- For example, activator RNAs stimulate transcription

- Enhancer-derived RNAs (eRNAs) fall into two categories

- 1D-eRNAs: Derived from enhancers that are transcribed unidirectionally.

- 2D-eRNAs: Derived from enhancers that are transcribed bidirectionally.

- More common and not polyadenylated. 
generally unspliced, with only the less common 1D-eRNAs undergoing polyadenylation.

In order to identify RA-associated loci where increased risk is likely to be mediated by lncRNA we have compiled a list of RA-associated regions that contain lincRNA and do not contain protein-coding genes. Regions of high linkage disequilibrium (encompassing all SNPs in linkage disequilibrium, $\mathrm{r}^{2}=0.8$ with the lead GWAS variant) were defined around the 102 regions associated with RA and computationally intersected with a database of lincRNA (using bedtools). ${ }^{15}$ This was performed with all lncRNAs annotated as intergenic in the IncRBase database. ${ }^{13}$ Of the 25 regions returned, 12 contained lncRNA that originate from Human Body Map RNA sequencing data, ${ }^{16}$ with the provenance of the remaining lncRNAs generally being Expressed Sequence Tags (ESTs) found in the NONCODE database. ${ }^{17}$ Manual curation, using UCSC genome browser, ${ }^{18}$ resulted in a list of 9 lncRNA containing RA regions that are sufficiently distant from protein-coding transcripts as to make any direct influence on their expression an unlikely consequence of disease-associated variants. A list of these regions is provided in table 1.

Fully characterising these lncRNA and the impact RA-associated variants have on their function will be an important area of research aimed at elucidating mechanisms of disease susceptibility. Generalised functional models for lncRNA are difficult to impose due to the variety of mechanisms observed, however, most functional lncRNA seem to act as scaffolds, regulating the formation of complexes that can be composed of protein, DNA and other RNA. ${ }^{14}{ }^{20}$ In brief, some of the complexes that lncRNA have been implicated in include the mediator complex ${ }^{21}$ and histone modifiers, such as Polycomb Repressive Complex 2 (PRC2). ${ }^{22}$ Frequently, lncRNA are implicated in regulating the transcription of other genes, in cis and in trans. ${ }^{23}$ Further complications include evidence that the act of transcription from certain lncRNA loci can be functional regardless of the transcript produced. ${ }^{24}$

Evidence supporting a role of lincRNAs in autoimmune disease is beginning to accumulate as this field attracts the interest of the scientific community. This evidence includes observations of differential regulation of lincRNA in pathways relevant to RA. For example in $\mathrm{CD} 14+$ monocytes isolated from patients with RA and stimulated with tumour-necrosis factor- $\alpha$ or interleukin 6 , two non-overlapping subsets of lincRNA were found to be differentially regulated (60 and 25 lincRNAs, respectively).$^{25} \mathrm{~A}$ separate study has linked changes in lincRNA expression to genotype using GWAS data, introducing the concept of lincRNA expression quantitative trait loci (lincRNA eQTLs). In general, these eQTLs were tissue specific and affected only lincRNAs and not a downstream regulated protein coding gene. ${ }^{26}$ As for enhancers, these findings encourage a stimulus and tissue-specific approach to investigating the role of lncRNA in RA.

\section{INVESTIGATING LNCRNA}

Many online resources are available that enable the prioritisation of candidate lncRNAs based on publicly available data (table 2). These include tools to overlay disease-associated SNPs with lncRNA, investigate the provenance and expression of these lncRNAs and to examine other genomic features of these loci, such as methylation, chromatin modifications and transcription factor binding.

Once genetically-associated lncRNA are identified and investigated bioinformatically, a wide variety of molecular biology techniques can be used to interrogate their function. In many cases the techniques are similar to those used to investigate the transcripts of coding genes, however, several additional considerations exist. ${ }^{34}$ It is important that experiments are designed in order to distinguish between loci that transcribe functional RNA, and loci where the process of transcription is important for the function of underlying regulatory elements. Perturbation experiments, including depletion and overexpression, are valuable for assessing RNA function, with genetic engineering a necessary component of experiments designed to investigate the role of genomic elements.

Table 1 Intergenic RA-associated regions that overlap lincRNA

\begin{tabular}{|c|c|c|c|c|c|}
\hline RA-associated region & Lead SNP & Gene & Expl. $\mathrm{H}^{2}$ & OR & Overlapping lincRNA \\
\hline chr1:38614865-38644861 & rs12140275 & POU3F1 & 0.039 & 1.11 & Inc-UTP11L-2 \\
\hline chr2:100806512-100835734 & rs9653442 & AFF3 & 0.064 & 1.11 & Inc-NMS-1 \\
\hline chr4:26085478-26128710 & rs11933540 & SMIM20 & 0.101 & 1.15 & Inc-RBPJ-1 \\
\hline chr6:137959233-138006504 & rs17264332 & TNFAIP3 & 0.100 & 1.17 & Inc-OLIG3-1 and Inc-TNFAIP3-3 \\
\hline chr6:159489789-159515309 & rs2451258 & TAGAP & 0.041 & 1.10 & Inc-FNDC1-7 \\
\hline chr8:102451261-102469182 & rs678347 & GRHL2 & 0.034 & 1.08 & Inc-ZNF706-5 \\
\hline chr8:129540462-129571140 & rs1516971 & TMEM75 & 0.044 & 1.15 & IncTMEM75-2 \\
\hline chr15:69984460-70010647 & rs8026898 & RPLP1 & 0.079 & 1.15 & Inc-RPLP1-2 \\
\hline chr16:86005836-86021627 & rs13330176 & IRF8 & 0.064 & 1.12 & Inc-IRF8-5 \\
\hline
\end{tabular}




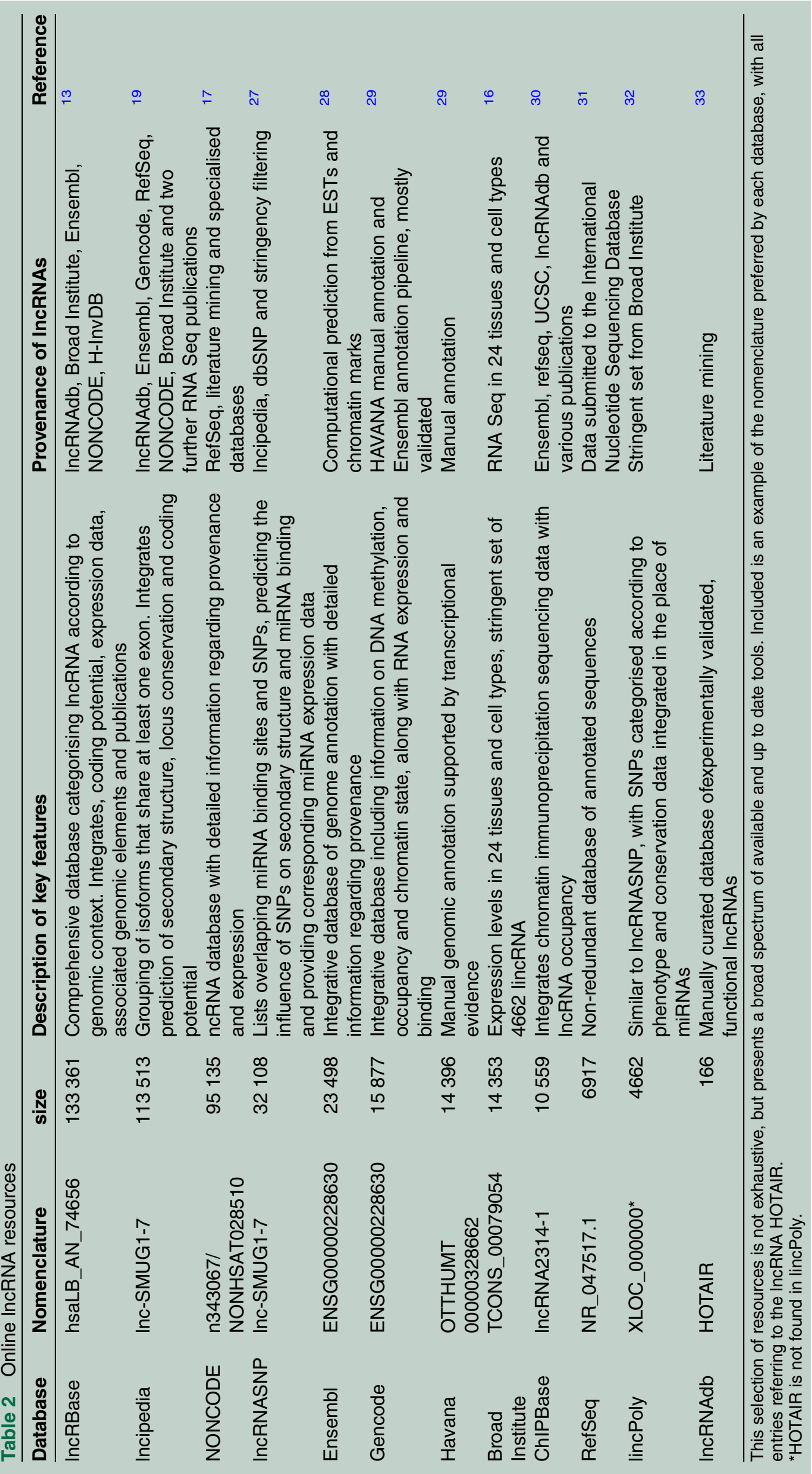


Table 3 Immunoprecipitation techniques for investigating IncRNA

\begin{tabular}{|c|c|c|c|}
\hline Acronym & Technique & Discriminating features & Reference \\
\hline CHART & $\begin{array}{l}\text { Capture hybridisation analysis of RNA } \\
\text { targets }\end{array}$ & $\begin{array}{l}\text { Tiled oligonucleotides against IncRNA, yielding bound } \\
\text { DNA and protein }\end{array}$ & 37 \\
\hline ChIRP & Chromatin isolation by RNA purification & $\begin{array}{l}\text { Pool of } 20 \text { nt probes and gluteraldehyde crosslinking. } \\
\text { Yields bound DNA and protein }\end{array}$ & 38 \\
\hline CLASH & $\begin{array}{l}\text { Crosslinking, ligation and sequencing of } \\
\text { hybrids }\end{array}$ & $\begin{array}{l}\text { Small pool of } \sim 20 \text { nt probes. Partial RNaseH treatment } \\
\text { during elution. Yields bound RNA }\end{array}$ & 39 \\
\hline iCLIP & $\begin{array}{l}\text { Individual nucleotide resolution } \\
\text { UV-crosslinking and immunoprecipitation }\end{array}$ & $\begin{array}{l}\text { Antibody used against protein of interest, with } \\
\text { interacting IncRNA nucleotide interrogated by } \\
\text { sequencing }\end{array}$ & 40 \\
\hline RAP & RNA antisense purification & $\begin{array}{l}\text { Large pool of } \sim 120 \text { nt probes. Yields bound DNA } \\
\text { and RNA }\end{array}$ & 4142 \\
\hline RIP & RNA immunoprecipitation & $\begin{array}{l}\text { Antibody used against protein of interest, with } \\
\text { presence of IncRNA interrogated. No cross-linking }\end{array}$ & 43 \\
\hline ChRIP & Chromatin RNA immunoprecipitation & $\begin{array}{l}\text { Antibody used against modified chromatin, with } \\
\text { presence of IncRNA interrogated }\end{array}$ & 44 \\
\hline ChOP & Chromatin oligo-affinity precipitation & Single $~ 80$ nt probe, yielding interacting DNA & 45 \\
\hline
\end{tabular}

Expression of lncRNAs can be investigated, as for any other transcript, by quantitative PCR (qPCR), microarray, RNA sequencing (RNA-Seq) or fluorescence in situ hybridisation (FISH). A modification of the protocol used to create probes for FISH can be used to simultaneously generate tools for depleting lncRNA expression. ${ }^{35}$ Commercial locked nucleic acids (LNAs) are also available for lncRNA depletion, with overexpression easily achieved using vector-based systems, similar to those used for protein-coding genes.

Several immunoprecipitation techniques exist that enable the identification of lncRNA-binding partners (examples of these techniques and references to their use are found in table 3). Given the propensity of lncRNAs to act as scaffolds, these experiments are of clear importance. When lncRNA are enhancer-derived, it is also wise to consider investigating their influence on DNA-DNA interactions. A variety of techniques exist for identifying and investigating enhancers and may well form the basis of further experiments. ${ }^{36}$

\section{POTENTIAL IMPACT}

This success in identifying a large number of genetic associations with RA is yet to be translated into a thorough understanding of its aetiology and pathogenesis. The challenge we now face is to pinpoint the disease-associated variants and understand how they dysregulate normal biology and lead to the development of disease processes (figure 1). One of the emerging concepts in post-GWAS studies is the discovery of an

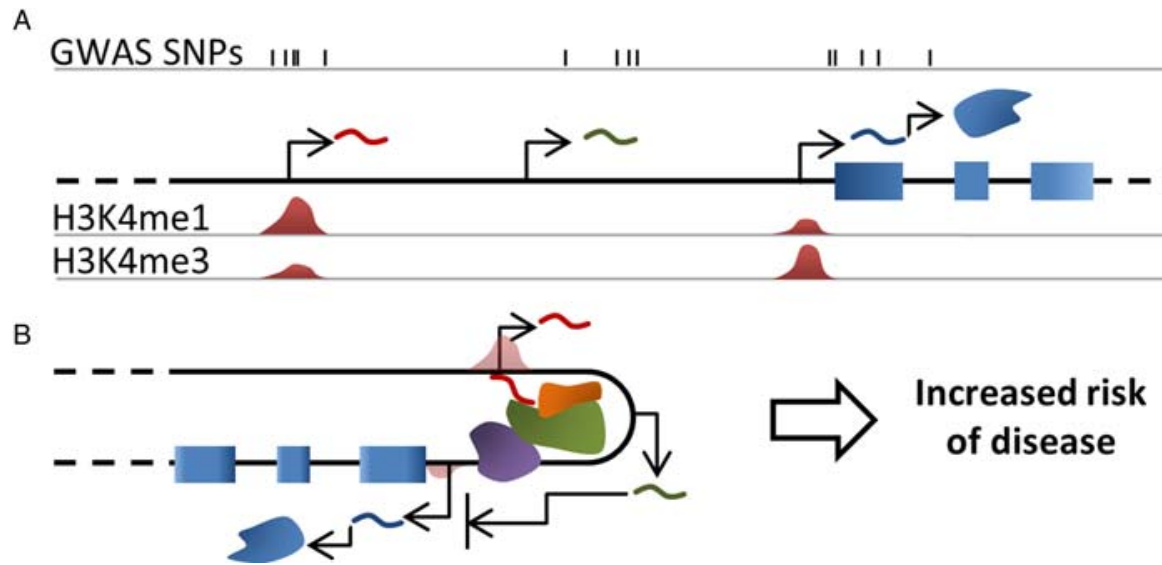

Figure 1 Graphical summary. (A) Genome-wide association studies single nucleotide polymorphism (GWAS SNPS) for complex diseases are mainly found outside of protein coding regions, and often overlay IncRNAs and/or enhancer elements. In this example, SNPs are found overlapping and proximal to three transcripts (curved lines); including two IncRNA, one of which is enhancer derived (red) and a mRNA (blue). (B) A detailed investigation of how these variants contribute to increased disease risk will have a profound impact on our understanding of the disease and is likely to implicate enhancers and IncRNA. IncRNAs (eg, green curved line) often function by regulating the transcription or translation of protein coding genes. One model of how this may occur is the mediation of chromatin interactions (eg, red curved line), which may involve the recruitment of chromatin modifiers, transcriptional activators or other proteins (green and orange forms, RNA pol II is represented by the purple form). 
enrichment of associated SNPs in intergenic regions, active in specific-cell types. We have described how bioinformatics analysis of RA loci implicates lncRNA and eRNA regions and propose that investigation of these loci may reveal novel insights into the pathogenesis of RA.

Thorough investigation of how disease-associated SNPs affect lncRNA, in a cellular and stimulation-specific manner, has the huge potential to link downstream genes, pathways and cellular outcomes empirically to disease causing variants. This is likely to implicate specific enhancer elements, regulatory targets and cell types in different diseases and will have an obvious impact on the understanding on complex disease aetiology, especially where these components are shared.

Contributors JD prepared the first draft. Re-draft was prepared by JW and SE. The structure of the review was developed equally by all authors.

Funding Arthritis Research UK.

Competing interests None declared.

Provenance and peer review Commissioned; externally peer reviewed.

Data sharing statement No additional data are available.

Open Access This is an Open Access article distributed in accordance with the terms of the Creative Commons Attribution (CC BY 4.0) license, which permits others to distribute, remix, adapt and build upon this work, for commercial use, provided the original work is properly cited. See: http:// creativecommons.org/licenses/by/4.0/

\section{REFERENCES}

1. Gregersen PK, Silver J, Winchester RJ. The shared epitope hypothesis. An approach to understanding the molecular genetics of susceptibility to rheumatoid arthritis. Arthritis Rheum 1987;30:1205-13.

2. Raychaudhuri S, Sandor C, Stahl EA, et al. Five amino acids in three HLA proteins explain most of the association between MHC and seropositive rheumatoid arthritis. Nat Genet 2012;44:291-6.

3. The Welcome Trust Case Control Consortium. Genome-wide association study of 14,000 cases of seven common diseases and 3,000 shared controls. Nature 2007;447:661-78.

4. Viatte S, Plant D, Raychaudhuri S. Genetics and epigenetics of rheumatoid arthritis. Nat Rev Rheumatol 2013:9:141-53.

5. Okada Y, Wu D, Trynka G, et al. Genetics of rheumatoid arthritis contributes to biology and drug discovery. Nature 2013;506:376-81.

6. Hindorff LA, Sethupathy P, Junkins HA, et al. Potential etiologic and functional implications of genome-wide association loci for human diseases and traits. Proc Natl Acad Sci USA 2009;106:9362-7.

7. Davison LJ, Wallace C, Cooper JD, et al. Long-range DNA looping and gene expression analyses identify DEXI as an autoimmune disease candidate gene. Hum Mol Genet 2012;21:322-33.

8. Musunuru K, Strong A, Frank-Kamenetsky M, et al. From noncoding variant to phenotype via SORT1 at the $1 \mathrm{p} 13$ cholesterol locus. Nature 2010;466:714-19.

9. Farh KK, Marson A, Zhu J, et al. Genetic and epigenetic fine mapping of causal autoimmune disease variants. Nature 2015;518:337-43

10. Hnisz D, Abraham BJ, Lee TI, et al. Super-enhancers in the control of cell identity and disease. Cell 2013;155:934-47.

11. Plank JL, Dean A. Enhancer function: mechanistic and genome-wide insights come together. Mol Cell 2014;55:5-14.

12. Bernstein BE, Birney E, Dunham I, et al. An integrated encyclopedia of DNA elements in the human genome. Nature 2012;489:57-74.

13. Chakraborty S, Deb A, Maji RK, et al. LncRBase: an enriched resource for IncRNA information. PLOS ONE 2014;9:e108010.

14. Nagano T, Fraser P. No-nonsense functions for long noncoding RNAs. Cell 2011;145:178-81.

15. Quinlan AR, Hall IM. BEDTools: a flexible suite of utilities for comparing genomic features. Bioinformatics 2010;26:841-2.

16. Cabili MN, Trapnell C, Goff L, et al. Integrative annotation of human large intergenic noncoding RNAs reveals global properties and specific subclasses. Genes Dev 2011;25:1915-27.
17. Xie C, Yuan J, Li H, et al. NONCODEv4: exploring the world of long non-coding RNA genes. Nucleic Acids Res 2014;42:D98-103.

18. Kent WJ, Sugnet CW, Furey TS, et al. The human genome browser at UCSC. Genome Res 2002;12:996-1006. doi:10.1101/gr.229102. Article published online before print in May 2002

19. Volders PJ, Helsens K, Wang X, et al. LNCipedia: a database for annotated human IncRNA transcript sequences and structures. Nucleic Acids Res 2013;41:D246-51.

20. Guttman M, Rinn JL. Modular regulatory principles of large non-coding RNAs. Nature 2012;482:339-46.

21. Lai $F$, Orom UA, Cesaroni M, et al. Activating RNAs associate with mediator to enhance chromatin architecture and transcription. Nature 2013;494:497-501.

22. Gupta RA, Shah N, Wang KC, et al. Long non-coding RNA HOTAIR reprograms chromatin state to promote cancer metastasis. Nature 2010;464:1071-6.

23. Vance KW, Ponting CP. Transcriptional regulatory functions of nuclear long noncoding RNAs. Trends Genet 2014;30:348-55.

24. Yoo EJ, Cooke NE, Liebhaber SA. An RNA-independent linkage of noncoding transcription to long-range enhancer function. $\mathrm{Mol}$ Cell Biol 2012;32:2020-9.

25. Müller N, Döring F, Klapper M, et al. Interleukin-6 and tumour necrosis factor- $\alpha$ differentially regulate lincRNA transcripts in cells of the innate immune system in vivo in human subjects with rheumatoid arthritis. Cytokine 2014;68:65-8.

26. Kumar V, Westra HJ, Karjalainen J, et al. Human disease-associated genetic variation impacts large intergenic non-coding RNA expression. PLoS Genet 2013;9:e1003201.

27. Gong J, Liu W, Zhang J, et al. IncRNASNP: a database of SNPs in IncRNAs and their potential functions in human and mouse. Nucleic Acids Res 2015;43(Database issue):D181-6.

28. Flicek P, Amode MR, Barrell D, et al. Ensembl 2014. Nucleic Acids Res 2014;42:D749-55.

29. Harrow J, Frankish A, Gonzalez JM, et al. GENCODE: the reference human genome annotation for The ENCODE Project. Genome Res 2012;22:1760-74.

30. Yang JH, Li JH, Jiang S, et al. ChIPBase: a database for decoding the transcriptional regulation of long non-coding RNA and microRNA genes from ChIP-Seq data. Nucleic Acids Res 2013;41(Database issue):D177-87.

31. Pruitt KD, Brown GR, Hiatt SM, et al. RefSeq: an update on mammalian reference sequences. Nucleic Acids Res 2014;42 (Database issue):D756-63.

32. Ning S, Wang P, Ye J, et al. A global map for dissecting phenotypic variants in human lincRNAs. Eur J Hum Genet 2013;21:1128-33.

33. Quek XC, Thomson DW, Maag JL, et al. IncRNAdb v2.0: expanding the reference database for functional long noncoding RNAs. Nucleic Acids Res 2015;43(Database issue):D168-73.

34. Bassett AR, Akhtar A, Barlow DP, et al. Considerations when investigating IncRNA function in vivo. Elife 2014;3:e03058.

35. Chakraborty D, Kappei D, Theis M, et al. Combined RNAi and localization for functionally dissecting long noncoding RNAs. Nat Methods 2012;9:360-2.

36. Shlyueva D, Stampfel G, Stark A. Transcriptional enhancers: from properties to genome-wide predictions. Nat Rev Genet 2014;15:272-86.

37. Simon MD, Wang $\mathrm{Cl}$, Kharchenko PV, et al. The genomic binding sites of a noncoding RNA. Proc Natl Acad Sci USA 2011;108:20497-502.

38. Chu C, Qu K, Zhong FL, et al. Genomic maps of long noncoding RNA occupancy reveal principles of RNA-chromatin interactions. Mol Cell 2011:44:667-78.

39. Kudla G, Granneman S, Hahn D, et al. Cross-linking, ligation, and sequencing of hybrids reveals RNA-RNA interactions in yeast. Proc Natl Acad Sci USA 2011;108:10010-15.

40. Huppertz I, Attig J, D'Ambrogio A, et al. iCLIP: protein-RNA interactions at nucleotide resolution. Methods 2014;65:274-87.

41. Engreitz JM, Sirokman K, McDonel P, et al. RNA-RNA interactions enable specific targeting of noncoding RNAs to nascent Pre-mRNAs and chromatin sites. Cell 2014;159:188-99.

42. Engreitz JM, Pandya-Jones A, McDonel P, et al. The Xist IncRNA exploits three-dimensional genome architecture to spread across the X chromosome. Science 2013;341:1237973.

43. Rinn JL, Kertesz M, Wang JK, et al. Functional demarcation of active and silent chromatin domains in human HOX loci by noncoding RNAs. Cell 2007;129:1311-23.

44. Pandey RR, Mondal T, Mohammad F, et al. Kcnq1ot1 antisense noncoding RNA mediates lineage-specific transcriptional silencing through chromatin-level regulation. Mol Cell 2008;32:232-46.

45. Mariner PD, Walters RD, Espinoza CA, et al. Human Alu RNA is a modular transacting repressor of mRNA transcription during heat shock. Mol Cell 2008;29:499-509. 